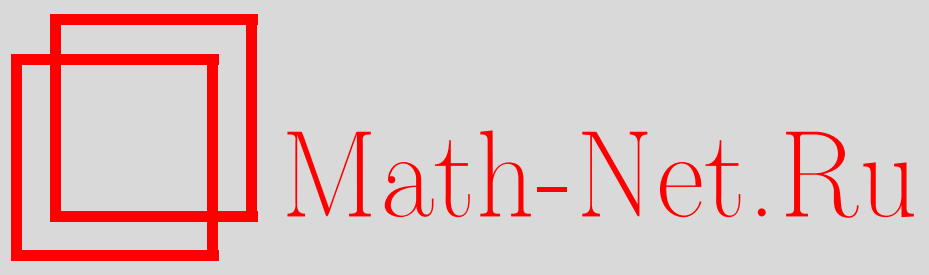

В. С. Владимиров, И. В. Волович, А. К. Гущин, Ю. Н. Дрожжинов, В. В. Жаринов, Б. И. Завьялов, В. А. Ильин, Г. И. Марчук, В. П. Михайлов, Е. И. Моисеев, С. М. Никольский, Б. В. Пальцев, Алексей Алексеевич Дезин (некролог), УМH, 2009, том 64, выпуск 3, 167173

DOI: https://doi.org/10.4213/rm9294

Использование Общероссийского математического портала Math-Net.Ru подразумевает, что вы прочитали и согласны с пользовательским соглашением http://www.mathnet.ru/rus/agreement

Параметры загрузки:

IP : 52.205 .19 .152

26 апреля 2023 г., 14:37:23

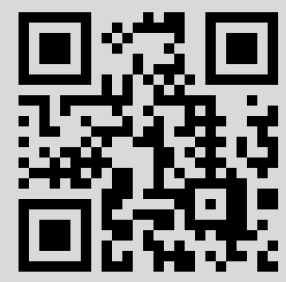




\section{Алексей Алексеевич Дезин}

4 марта 2008 г. на 85-м году ушел из жизни известный ученый-математик, крупный специалист в области дифференциальных уравнений с частными производными, функционального анализа, математической физики, научный сотрудник Математического института им. В.А. Стеклова РАН, профессор Московского физико-технического института, а затем Московского государственного университета им. М.В. Ломоносова, лауреат Государственной премии СССР Алексей Алексеевич Дезин.

А. А. Дезин родился 23 апреля 1923 г. в Москве в семье служащего. В силу сложившихся в те годы обстоятельств свою трудовую деятельность он начал рано - после девяти классов школы. В суровые годы Великой Отечественной войны Алексей Алексеевич в рядах Советской Армии участвовал в войне с Японией. После окончания войны и демобилизации окончил вечернюю

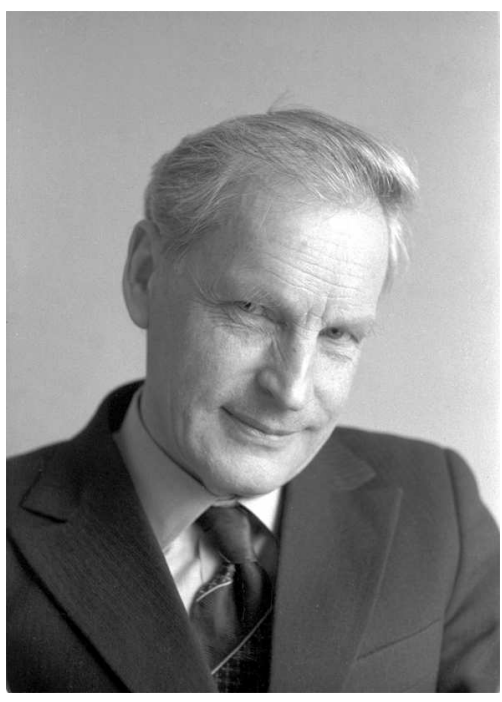
школу. В 1948 г. он становится студентом механико-математического факультета МГУ, который с отличием оканчивает в 1953 г. В том же году А. А. Дезин поступает в аспирантуру мехмата МГУ. Его научным руководителем в студенческие годы и в аспирантуре был академик С. Л. Соболев.

В 1956 г., после окончания аспирантуры, А. А. Дезин начал педагогическую деятельность на кафедре высшей математики Московского физико-технического института. В этом же году он защитил кандидатскую диссертацию "О граничных задачах для линейных систем уравнений с частными производными первого порядка". В 1957 г. Алексей Алексеевич перешел на основную работу в Математический институт им. В.А. Стеклова АН СССР, в котором работал до последних дней в отделе математической физики. При этом его научно-педагогическая деятельность долгие годы оставалась связанной с МФТИ, в котором он работал более 30 лет по совместительству - он был профессором кафедры математической физики, возглавлявляемой академиком А. А. Дородницыным. С 1994 г. до последних дней жизни А. А. Дезин являлся по совместительству профессором кафедры общей математики факультета ВМК МГУ, возглавляемой академиком В. А. Ильиным.

Первые научные работы Алексея Алексеевича были посвящены вопросам продолжения функций, теоремам вложения, а также исследованию условий разрешимости 2-й краевой задачи для полигармонического уравнения. При этом уже в дипломной работе он развивал технику операторов осреднения переменного радиуса [1], которая и по настоящее время остается эффективным аппаратом в теории продолжения функций, в теории граничных задач (при исследовании проблемы совпадения слабых и сильных решений). 
В цикле работ А. А. Дезина, выполненном в основном до 1959 г. и включающем кандидатскую диссертацию [3]-[6], изучались граничные задачи для линейных систем с частными производными 1-го порядка. Им была начата разработка метода энергетических неравенств для исследования разрешимости смешанных задач в гиперболическом случае. А.А. Дезиным, в одно время с К. Фридрихсом, был введен и исследовался весьма важный для приложений класс систем 1-го порядка, получивший в дальнейшем название симметричных положительных систем. Им были получены новые результаты по классификации и по корректной разрешимости некоторых естественных типов граничных задач для изучаемых систем, было найдено условие существования разрешимого расширения ("корректной краевой задачи") для линейных систем 1-го порядка с постоянными коэффициентами. Наряду с этим А. А. Дезиным развивались "функциональные методы" и для некоторых уравнений второго порядка, в частности, им были установлены существование и единственность обобщенных решений смешанных задач для уравнений волнового и теплопроводности.

Важным и ярким этапом, характеризующим творчество Алексея Алексеевича, явился следующий, выполненный в период с 1958 по 1962 г. цикл работ [7]-[10], посвященный инвариантным системам с частными производными на многообразиях. Стремясь понять структуру системы Коши-Римана, а также известной системы Мойсила-Теодореску в $\mathbb{R}^{3}$, А. А. Дезин получил обобщение таких эллиптических систем 1-го порядка в терминах дифференциальных форм для случая произвольного $n$-мерного гладкого риманова многообразия и нашел их завершенное и простое выражение через оператор $d$ внешнего дифференцирования и метрически сопряженный с ним оператор $\delta$. Такие системы действительно по ряду принципиальных свойств являются точными аналогами классической системы Коши-Римана.

Многомерные системы Коши-Римана исследовались А. А. Дезиным не только на замкнутых римановых многообразиях. Он рассмотрел для них также и задачи на многообразиях с краем с однородными краевыми условиями либо только для нормальных составляющих, либо только для касательных составляющих дифференциальной формы $\omega$. С помощью процедуры "подклеивания дубля" и надлежащих продолжений формы $\omega$ на дубль он свел такие задачи к системам на замкнутом многообразии. В результате для рассмотренных краевых задач была установлена нётеровость и найдены формулы индекса, очень просто выражаемые через эйлерову характеристику многообразия.

В этом цикле были рассмотрены также и некоторые другие инвариантные системы. А. А. Дезин показал, как осуществить "правильный" переход от эллиптического случая к гиперболическому и к параболическому. Им были найдены инвариантные записи (через операторы $d$ и $\delta$ ) также и некоторых систем, встречающихся в математической физике. Были доказаны существование и единственность обобщенных решений задачи типа Гурса для гиперболических систем в многомерном случае, смешанной задачи для параболических систем, а также некоторых других краевых задач. Большая часть из этих результатов и ряд других вошли в докторскую диссертацию Алексея Алексеевича [9] "Инвариантные дифференциальные операторы и граничные задачи", защищенную им в 1961 г. и опубликованную затем отдельным томом [10] в "Трудах МИАН".

В течение многих лет, начиная с 1962 г., А.А. Дезин активно проводил исследования ряда принципиальных проблем для линейных уравнений с частными производными на специальном классе модельных операторных уравнений. Здесь его в первую очередь интересовали вопросы реализации разрешимых расширений дифференциальных операторов с помощью конкретных граничных условий. Для случая модельных уравнений им было введено близкое к понятию разрешимого расширения понятие "правильного" оператора, порождаемого общей дифференциальной операцией с постоянными коэффициентами в ограниченной области, изучалась возможность описания правильных операторов соответствующими граничными условиями. Эти 
исследования отличают привлечение спектральной теории для общего описания правильно поставленных граничных задач, а также систематическое использование нелокальных граничных условий. Он дал примеры уравнений с частными производными, для которых не существует корректных задач с локальными граничными условиями. См. [12], [13], [15].

В рамках модельных операторных уравнений А. А. Дезиным были получены, например, следующие результаты для неклассических уравнений с частными производными: им были найдены правильно поставленные задачи для ультрагиперболического и псевдопараболического уравнений [11] (для ультрагиперболического уравнения проблема отыскания корректно поставленной граничной задачи была сформулирована академиком И. Г. Петровским еще в 1946 г.). Для модельных операторных уравнений 1-го и 2-го порядка он получил исчерпывающее описание правильных расширений.

В тех же рамках А. А. Дезин изучал и некоторые качественные вопросы теории уравнений с частными производными. Для операторных уравнений с разрывными коэффициентами он предложил классификацию различных типов иррегулярностей. При исследовании граничных задач для уравнений со степенным вырождением им было получено [30], [31] детальное описание характера выполнения граничных условий на линии вырождения, причем в ситуациях, оставшихся недостаточно изученными в известных работах по вырождающимся эллиптическим уравнениям. Здесь следует также отметить работы А. А. Дезина, посвященные изучению изменения точечного спектра некоторых несамосопряженных операторов с частными производными при малых возмущениях главной части [43], и, кроме того, работы по специальным нелинейным моделям, содержащим нелокальные граничные условия [45], [46].

Большой интерес для А. А. Дезина всегда представляли и актуальные задачи гидродинамики, к которым он часто обращался в своих исследованиях. Им были найдены новые инвариантные формы записи уравнений Эйлера. Он получил ряд интересных результатов по проблеме образования циркуляционных зон течений идеальной жидкости, им был предложен метод исследования структурных свойств эйлеровых векторных полей, основанный на изучении специальных свойств однопараметрических семейств течений. Для одной линеаризованной модели течения вязкой несжимаемой жидкости А. А. Дезиным была установлена регулярность вырождения при исчезающей вязкости. См. [16], [27], [28], [32].

Результаты этого цикла были систематически изложены в двух монографиях А. А. Дезина: "Общие вопросы теории граничных задач" [29] и "Дифференциально-операторные уравнения. Метод модельных операторов в теории граничных задач" [51]. За первую из этих монографий Алексею Алексеевичу в 1988 г. была присуждена Государственная премия СССР.

Большой цикл работ А.А. Дезина посвящен моделированию объектов анализа и физики на дискретных структурах. Известная в топологии связь между комбинаторным и континуальным определением кольца когомологий привела его к идее построения внутренним образом определенных дискретных моделей инвариантных дифференциальных операторов. Алексеем Алексеевичем был развит подход, который оказался хорошо приспособленным для получения разностных аналогов операторов $d$ и $\delta$ и, в частности, операторов векторного анализа. Этот подход вызвал значительный интерес и нашел применение в теории разностных схем для уравнений математической физики. См. [34], [41], [42], [44].

Такого рода модели А. А. Дезин изучал и для специального класса эллиптических систем. Им были получены дискретные аналоги теорем Ходжа и Кодаиры об ортогональных разложениях. С помощью конструкции типа спенсеровской исследовалась глобальная разрешимость систем разностных уравнений, изучались задачи, являющиеся аналогами граничных [18]. Кроме того, для некоторых разностных операторов на простейших областях были проведены исследования характера изменения спектра при малых вариациях области. В данном цикле следует также выделить группу 
работ, в которых на простейших конечных структурах прослеживались принципиальные черты перехода от классического к квантово-механическому описанию объекта и от квантовой механики - к теории поля [21], [22].

Эти исследования составили основу монографии А. А. Дезина "Многомерный анализ и дискретные модели" [44]. К этому циклу примыкает и серия недавних работ Алексея Алексеевича. Первые из них - работы по двумерным моделям электродинамики и ее обобщениям. Последние работы посвящены анализу дедуктивной структуры квантовой механики, использующему как континуальные, так и дискретные модели физического пространства. Были рассмотрены варианты "вывода" уравнений Шрёдингера, Гейзенберга и Клейна-Гордона, опирающиеся на те или иные постулаты. Проанализировано различие предположений: "время-параметр" и "время-элемент структуры". Выяснено принципиальное отличие динамики Фейнмана [46]-[50].

А. А. Дезин - автор более 80 научных публикаций и 4 монографий, переведенных на английский и немецкий языки. Научное творчество Алексея Алексеевича отличает прежде всего оригинальность, глубокое проникновение в суть изучаемых проблем, начиная с достаточно простых моделей, выявление фундаментальных связей с другими областями математики, а также с физикой, многогранность, постоянное стремление к новым подходам, к постановке и изучению новых задач. Оно оказало и оказывает свое влияние на развитие ряда разделов общей теории уравнений с частными производными и граничных задач, а также некоторых других областей.

А.А. Дезин постоянно вел большую научно-педагогическую работу. Много сил и времени он отдавал подготовке молодых специалистов, всегда был заботливым научным руководителем. Среди его учеников 7 докторов наук. В течение многих лет он читал для студентов МФТИ и ВМК МГУ оригинальный, насыщенный нетрадиционным материалом курс "Дополнительные главы теории уравнений в частных производных", был одним из руководителей научных семинаров в МИРАН и на кафедре общей математики ВМК МГУ. Он много лет являлся членом специализированных советов по защите диссертаций, был членом редколлегий ряда научных журналов, членом экспертной группы ВАК СССР.

Как человека Алексея Алексеевича отличали такие замечательные качества, как неизменная благожелательность, доброта, отзывчивость, скромность, широкая эрудиция, разносторонность интересов. Для нас - его коллег, друзей, учеников - его уход из жизни - невосполнимая потеря. Светлый его образ, память о нем навсегда останутся в наших сердцах.

В.С. Владимиров, И.В. Волович, А.К. Гущин, Ю.Н. Дрожжинов, В.В. Жаринов, Б.И. Завъллов, В.А. Илъин, Г. И. Марчук, В.П. Михайлов, Е.И. Моисеев, С. М. Никольский, Б. В. Палъцев

\section{Список избранных научных работ А. А. Дезина}

[1] "К теоремам вложения и задаче о продолжении функций”, Докл. АН CCCP, 88:5 (1953), 741-743.

[2] "Вторая краевая задача для полигармонического уравнения в пространстве $W_{2}^{(m) ", ~ Д о к л . ~ А Н ~ С С С Р, ~ 96: 5 ~(1954), ~ 901-903 . ~}$

[3] "Смешанные задачи для некоторых симметричных гиперболических систем", Докл. АН СССР, 107:1 (1956), 13-16.

[4] О граничных задачах для линейных систем уравнений с частными производными первого порядка, Дис. ... канд. физ.-матем. наук, МГУ, М., 1956.

[5] “Теоремы существования и единственности решений граничных задач для уравнений с частными производными в функциональных пространствах", УМH, 14:3 (1959), 21-73; англ. пер.: "Existence and uniqueness theorems for boundary value 
problems for partial differential equations in function spaces", Amer. Math. Soc. Transl. Ser. 2, 42 (1964), 71-128.

[6] "Граничные задачи для некоторых симметричных линейных систем первого порядка", Матем. сб., 49:4 (1959), 459-484.

[7] "Инвариантные эллиптические системы уравнений", Сиб. матем. журн., 1:4 (1960), 578-608.

[8] “Инвариантные дифференциальные операторы и граничные задачи”, УМH, 16:2 (1961), 160-162.

[9] Инвариантные дифференциалъные операторы и граничные задачи, Дис. ... докт. физ.-матем. наук, Новосибирск, СО АН СССР, 1961.

[10] "Инвариантные дифференциальные операторы и граничные задачи", Тр. МИАН, 68, Наука, М., 1962, 1-88.

[11] "Простейшие разрешимые расширения для ультрагиперболического и псевдопараболического операторов”, Докл. АН СССР, 148:5 (1963), 1013-1016; англ. пер.: "The simplest solvable extensions of ultrahyperbolic and pseudoparabolic operators", Soviet Math. Dokl., 4 (1963), 208-211.

[12] "О разрешимых расширениях для дифференциальных операторов с частными производными", Материалы к совместному советско-американскому Симпозиуму по уравнениям с частными производными, СО АН СССР, Новосибирск, 1963, 65-66.

[13] “Операторы с первой производной по 'времени' и нелокальные граничные условия", Изв. АН СССР. Сер. матем., 31:1 (1967), 61-86; англ. пер.: "Operators involving a first derivative with respect to time and nonlocal boundary conditions", Izv. Math., 1:1 (1967), 57-79.

[14] "О методе ортогональных разложений", Сиб. матем. журн., 9:5 (1968), 1062-1074; англ. пер.: "Method of orthogonal expansions", Siberian Math. J., 9:5 (1968), 788-797.

[15] "Несуществование некоторых разрешимых расширений”, Докл. АН CCCP, 183:3 (1968), 507-510; англ. пер.: "Nonexistence of certain solvable extensions", Soviet Math. Dokl., 9 (1968), 1400-1403.

[16] "Некоторые модели, связанные с уравнениями Эйлера", Дифферени. уравнения, 6:1 (1970), 17-26.

[17] "Неклассические граничные задачи”, Дифференииалъные уравнения с частными производными, Наука, М., 1970, 81-95 (совм. с В. Н. Масленниковой).

[18] "Глобальная разрешимость многомерных разностных уравнений и конструкция типа спенсеровской", Докл. АН СССР, 196:1 (1971), 28-31; англ. пер.: "Global solvability of multidimensional difference equations and a construction of Spencer type", Soviet Math. Dokl., 12 (1971), 25-29.

[19] "О спектре некоторых разностных операторов", Сиб. матем. журн., 13:1 (1972), 86-93; англ. пер.: "The spectrum of certain difference operators", Siberian Math. J., 13:1 (1972), 61-66.

[20] "Естественные дифференциальные операторы и разделение переменных", Дифферени. уравнения, 14:9 (1973), 25-31.

[21] "Об одной модели квантования", Сиб. матем. журн., 14:1 (1973), 44-52; англ. пер.: "A model of quantization", Siberian Math. J., 14:1 (1973), 29-35.

[22] “О конечных моделях в квантовой теории поля”, Сиб. матем. журн., 14:6 (1973), 1216-1230; англ. пер.: "Finite models in quantum field theory", Siberian Math. J., 14:6 (1973), 853-863. 
[23] "Комбинаторная модель евклидова пространства и разностные операторы", Cиб. матем. журн., 16:4 (1975), 700-713; англ. пер.: "Combination model of Euclidean space and difference operators", Siberian Math. J., 16:4 (1975), 536-545.

[24] "К общей теории граничных задач", Матем. сб., 100:2 (1976), 171-180; англ. пер.: "On the general theory of boundary value problems", 29:2 (1976), 147-155.

[25] "Операторные уравнения второго порядка", Сиб. матем. журн., 19:5 (1978), 1032-1042; англ. пер.: "Second-order operator equations", Siberian Math. J., 19:5 (1978), 730-737.

[26] "О слабых и сильных расширениях дифференциальных операторов", Матем. заметки, 24:5 (1978), 687-698.

[27] “О некоторых системах уравнений, содержащих малый параметр", Матем. сб., 111:3 (1980), 323-333; англ. пер.: "On some systems of equations containing a small parameter", Math. USSR-Sb., 39:3 (1981), 289-298.

[28] "О некоторых математических задачах в гидродинамике", Дифференциальные уравнения с частными производными (Новосибирск, 1978), Наука, Новосибирск, 1980, 21-31 (совм. с Т. И. Зеленяком, В.Н.Масленниковой).

[29] Общие вопросы теории граничных задач, Наука, М, 1980.

[30] "Вырождающиеся операторные уравнения", Матем. сб., 115:3 (1981), 323-336; англ. пер.: "Degenerate operator equations", Math. USSR-Sb., 43:3 (1982), 287-298.

[31] "О слабой и сильной иррегулярности”, Дифферени. уравнения, 17:10 (1981), 1851-1858; англ. пер.: "On weak and strong irregularity", Differ. Equ., 17:10 (1982), 1160-1165.

[32] "Инвариантные формы и структурные свойства гидродинамических уравнений Эйлера", Z. Anal. Anwend., 2:5 (1983), 401-409.

[33] "О переопределенных граничных задачах", Сиб. матем. журн., 24:5 (1983), 43-47; англ. пер.: "Nonperiodic boundary problems", Siberian Math. J., 24:5 (1983), $681-685$.

[34] “Дискретные модели в математической физике”, Актуальные проблемы математической физики и вычислительной математики, Наука, М., 1984, 75-89.

[35] Уравнения, операторы, спектры, Знание, М., 1984.

[36] "Спектральные характеристики общих граничных задач для оператора $D^{2}$ ", $M a$ тем. заметки, 37:2 (1985), 249-256; англ. пер.: "Spectral characteristics of general boundary-value problems for operator $D^{2}$ ", Math. Notes, 37:2 (1985), 142-146.

[37] "О задачах для линейных дифференциальных операций”, Матем. сб., 129:3 (1986), 397-406; англ. пер.: "On problems for linear differential operations", Math. USSS-Sb., 57:2 (1987), 411-419.

[38] "Нестандартные задачи", Матем. заметки, 41:3 (1987), 356-364; англ. пер.: "Nonstandard problems", Math. Notes, 41:3 (1987), 205-210.

[39] Двумерные преобразование Абеля и приложения, Изд-во Тбилисского ун-та, Тбилиси, 1987.

[40] "Уравнения в частных производных", Математическая физика и комплексный анализ, Тр. МИАН, 176, М., Наука, 1987, 259-299 (совм. с А. В. Бицадзе, В. С. Виноградовым, В.А.Ильиным); англ. пер.: "Partial differential equations", Mathematical physics and complex analysis, Proc. Steklov Inst. Math., № 3, 1988, 263-300 (with A. V. Bitsadze, V.S. Vinogradov, V. A Il'in).

[41] "Дискретные аналоги соотношений математической гидродинамики”, Cuб. матем. журн., 29:5 (1988), 97-103; англ. пер.: "Discrete analogs of equations in mathematical hydrodynamics", Siberian Math. J., 29:5 (1988), 765-770. 
[42] "Сплайн-формы, разностные уравнения, аппроксимация", Дифферени. уравнения, 24:1 (1988), 32-43; англ. пер.: "Spline-forms, difference equations, approximation", Differ. Equ., 24:1 (1988), 24-33.

[43] "О возмущениях точечного спектра", Дифферени. уравнения, 26:1 (1990), 23-30; англ. пер.: "Perturbations of the point spectrum", Differ. Equ., 26:1 (1990), 20-25.

[44] Многомерный анализ и дискретные модели, Наука, М., 1990; англ. пер.: Multidimensional analysis and discrete models, CRC Press, Boca Raton, FL, 1995.

[45] "О простейших нелинейных задачах", Дифферени. уравнения, 28:1 (1992), 52-59; англ. пер.: "On elementary nonlinear problems", Differ. Equ., 28:1 (1992), 50-57.

[46] "Возмущение условий периодичности в простейшей нелинейной модели", Докл. PAH, 323:6 (1992), 1012-1015 (совм. с Н. Г. Марчуком); англ. пер.: "Perturbation of periodicity conditions in an elementary nonlinear model", Russian Acad. Sci. Dokl. Math., 45:2 (1992), 467-469 (with N. G. Marchuk).

[47] "Модели, порождаемые уравнениями Янга-Милса", Дифферени. уравнения, 29:5 (1993), 846-851; англ. пер.: "Models generated by Yang-Mills equations", Differ. Equ., 29:5 (1993), 724-728.

[48] "Об уравнениях Шрёдингера и Клейна-Гордона”, Дифферени. уравнения, 30:6 (1994), 1023-1033; англ. пер.: "On the Schrödinger and Klein-Gordon equations", Differ. Equ., 30:6 (1994), 948-957.

[49] "Уравнение Шрёдингера и динамика Фейнмана", Дифферени. уравнения, 33:1 (1997), 96-106; англ. пер.: "The Schrödinger equation and Feynman dynamics", Differ. Equ., 33:1 (1997), 97-107.

[50] “Об уравнениях Шрёдингера и Клейна-Гордона. II”, Дифферени. уравнения, 34:5 (1998), 789-798; англ. пер.: "On the Schrödinger and Klein-Gordon equations. II", Differ. Equ., 34:6 (1998), 786-795.

[51] Дифференииально-операторные уравнения. Метод модельных операторов в теории граничных задач, Тр. МИАН, 229, М., Наука, 2000; англ. пер.: Operatordifferential equations. The method of model operators in the theory of boundary value problems, Proc. Steklov Inst. Math., 2, 2000. 